\title{
Circuit
}

Musiques contemporaines

\section{Père Louis Hage, grand spécialiste du chant maronite}

\section{Badih El Hajj}

Volume 29, numéro 3, 2019

URI : https://id.erudit.org/iderudit/1066491ar

DOI : https://doi.org/10.7202/1066491ar

Aller au sommaire du numéro

\section{Éditeur(s)}

Circuit, musiques contemporaines

\section{ISSN}

1183-1693 (imprimé)

1488-9692 (numérique)

Découvrir la revue

Citer ce document

El Hajj, B. (2019). Père Louis Hage, grand spécialiste du chant maronite. Circuit, 29(3), 99-101. https://doi.org/10.7202/1066491ar d'utilisation que vous pouvez consulter en ligne.

https://apropos.erudit.org/fr/usagers/politique-dutilisation/ 


\section{Père Louis Hage, grand spécialiste du chant maronite}

Père Badih El Hajj

Je crois à la musique maronite traditionnelle et à sa pérennité. - P. Louis Hage'.

C'est à Paris, lors de la préparation de ma thèse de doctorat en musicologie, que j’ai eu la chance de rencontrer la compositrice canadienne Katia Makdissi-Warren. Elle était alors venue spécialement d'Allemagne pour apprendre les musiques syriaques et arabes auprès du grand maître de la musique maronite, le père Louis Hage (1938-2010)2. À cette époque, Hage fut élu à titre d'Assistant général de l'Ordre libanais maronite (OLM). Ces nouvelles responsabilités l'obligèrent à quitter Paris pour le Liban, et il me confia la mission d'enseigner la musique orientale à $\mathrm{M}^{\mathrm{me}}$ Makdissi-Warren. Enchanté d'être l'un des disciples de P. Louis Hage et honoré par son choix, je me suis lancé dans cette mission, heureux de faire la connaissance d'une véritable artiste: non seulement musicienne et compositrice, mais aussi personne talentueuse, modeste et cultivée. Les premiers cours portaient sur l'étude d'un instrument oriental, le oud, et sur la pratique des maqamats et de l'improvisation taqasim.

Je présente dans cet article le P. Louis Hage, une personne qui a contribué à la formation de la personnalité musicale de $\mathrm{M}^{\mathrm{me}}$ Makdissi-Warren. Après deux années d'études avec Hage, Makdissi-Warren a composé des œuvres métissant les différentes musiques.

\section{Père Louis Hage, homme de culture}

Musicologue de renom, P. Louis Hage était un grand spécialiste du chant maronite et l'un des piliers de la réforme liturgique et musicale de l'Église maronite. Hage possédait une culture vaste et profonde, ayant obtenu une licence en théologie à Rome en 1966; un doctorat en musicologie à la Sorbonne, à Paris, en 1970; un diplôme de l'École pratique des hautes études à Paris en 1971; un doctorat d'État en 1980 (équivalence par le ministère de l'Éducation nationale) au Liban; ainsi qu'un doctorat honoris causa ès lettres du Stonehill College au Massachusetts en 1990. Ce parcours scolaire témoigne également de sa maîtrise de l'arabe, du latin, du syriaque, du français, de l'italien, de l'anglais et de l'allemand. Se débrouillant également en grec et en hébreu, ce polyglotte a utilisé avec aisance l'une ou l'autre de ces langues dans ses livres, articles et conférences. Par la polyvalence de ses recherches, la musicologie acquiert ses lettres de créance. Avec les pratiques traditionnelles d'instruments et de chant que cette discipline implique, en plus des compétences didactiques qui en découlent, elle s'élève, grâce au P. Louis Hage, au rang de discipline universitaire bien nourrie.

En tant que pédagogue, Hage cherchait à partager avec ses étudiants certaines approches scientifiques indispensables à la conception et à la réalisation de leurs projets. Pour lui, l'enseignement n'était pas seulement une transmission de connaissances, mais aussi l'accomplissement d'une vocation. Pour cela, j’estime que le choix de Katia Makdissi-Warren d'aller vers 
Hage reposait sur la reconnaissance en lui de l'âme et des vertus d'un grand maître du chant maronite et de la musique arabe.

En lien avec la musicologie, le P. Louis Hage a fondé, en 1970, l'Institut de musicologie à l'Université Saint-Esprit de Kaslik (USEK). Cet institut est devenu une Faculté de musique en 1993, et Hage en est resté le doyen pendant plusieurs années. Sous sa tutelle, la faculté a pris une envergure internationale, et formé des étudiants provenant du Liban et de différents pays arabes.

\section{Le P. Louis Hage et le chant maronite}

Compositeur, artiste, écrivain, poète, visionnaire et savant, le P. Louis Hage a laissé un apport culturel riche et varié. Ses nombreuses tâches universitaires, administratives, religieuses et musicales ne l'ont pas empêché d'effectuer, inlassablement, des recherches et des publications littéraires et musicales qui ont constitué le fondement d'une bibliothèque vaste et bien nantie. L'objet principal de ses œuvres fut le chant de l'Église maronite. Il consacra plusieurs volumes à l'étude de ce chant, à son répertoire, son analyse, ses références, sa forme, sa structure et sa classification; il ajoute encore à cet ensemble un précis de chant maronite publié en français, en anglais et en arabe.

Spécialiste du chant maronite, Hage n'a cessé, tout au long de sa vie, de protéger, d'améliorer et de propager la vraie musique maronite sous tous les cieux. Il était le gardien de son authenticité - qui a traversé les âges - et de sa tradition - qui ne se renouvelle que par la foi et l'attachement aux origines. Il luttait contre les velléités superficielles qui perturbent, depuis quelques décennies, la marche des croyants. Il a su chanter sa mission sur des airs spirituels, dont l'écho résonnera à jamais à travers les âges et les générations. Sa réputation dans le domaine de la musique sacrée a fait de lui une référence. Ses écrits et ses œuvres si prisés en sont la preuve.
Qu'est-ce que le chant maronite? Le chant maronite est le chant sacré de l'Église antiochienne des Maronites. Le répertoire des chants actuellement en usage dans l'Église maronite est très varié. À partir de 1970, Hage a de nombreuses fois noté les mélodies maronites traditionnelles dans des livres et livrets liturgiques parus entre 1972 et 1982. Il n'est pas inutile d'ajouter que ces chants ont eu un très grand succès (messes, concerts, disques) au Liban comme à l'étranger. Des communautés et des paroisses chantent désormais ces mélodies qui tombaient lentement dans l'oubli avec le texte syriaque lui-même, au profit de quelques nouveaux chants faciles. Pour Hage, «les chants traditionnels sont des reliques sanctifiées par les lèvres de saints ancêtres qui ne cessaient nuit et jour de les chanter et de les offrir au Seigneur en sacrifice de louange ${ }^{3}$.»

L'expérience du P. Hage et ses recherches, tant sur le chant syriaque en général que sur le chant maronite en particulier, lui ont permis de projeter une collection en plusieurs volumes consacrée à ce chant. La collection «Musique maronite ${ }^{4}$ » intéresse aussi bien les fidèles maronites qu'un bon nombre d'amateurs et de musicologues étrangers, car elle met entre leurs mains des instruments de travail nécessaires à toute recherche approfondie. On peut y repérer cinq groupes bien distincts quant à leur origine, leur nature et leur valeur traditionnelle et liturgique. En outre, chaque groupe est susceptible d'être divisé en d'autres couches et catégories.

Hage fut l'un des piliers de la réforme liturgique et musicale qui eut lieu à l'USEK, en 1970. Depuis 1976 se sont succédé, à un rythme serré, des publications liturgiques munies de notations musicales et accompagnées d'icônes, de disques et de cassettes. Les chants composés à cette époque ont servi à enrichir le missel officiel publié par la Commission liturgique du patriarcat maronite. 


\section{Conclusion}

Tout au long de sa carrière, le P. Louis Hage s'est révélé être un homme de principes et de valeurs. Noble, croyant, sensible et réservé, il fut tout autant reconnu pour son caractère ferme, décisif et déterminé.

Merci, Katia Makdissi-Warren, de votre gratitude envers mon confrère et mon maître, le père et professeur Louis Hage. Et félicitations pour vos belles compositions qui transportent des échos de notre Orient chéri.

\section{B I B L I O GRAPH IE}

HaGE, Louis (1971-1997), Musique maronite: collection en 7 volumes portant sur plusieurs domaines de la musique utilisée dans l'Église maronite d'Antioche, Kaslik, Université Saint-Esprit de Kaslik.

Hage, Louis (2005), Écrits musicaux, Kaslik, Université Saint-Esprit de Kaslik.
1. Citation tirée du mot de P. Louis Hage prononcé durant la cérémonie, à l'occasion de la présentation de son VIII ${ }^{\mathrm{e}}$ volume de la collection "Musique Maronite" à Kaslik (Liban) en mars 1995. Cette citation apparaît dans Hage, 2005, p. 108.

2. Le Père Louis Hage (1938-2010), moine et prêtre de l'Ordre libanais maronite, a été à la base de la réforme et de la codification de la musique sacrée de l’Église syriaque maronite.

3. Louis Hage, Musique maronite, Le chant maronite, tome II, Kaslik, Bibliothèque de l'Université Saint-Esprit, $n^{0}$ 26, 1995, p. 19.

4. Collection de sept volumes en dix tomes portant sur plusieurs domaines de la musique en usage dans l'Église maronite d'Antioche. Série mélodies: vol. 1, Le chant maronite, tome I (1971); vol. 2, Le chant maronite, tome II (1994); vol. 3A, Monuments du chant maronite, tome I (1990); vol. 3B, Monuments du chant maronite, tome II (1991); vol. 4A, Analyse, classement et références, tome I (1990); vol. 4B, Analyse, classement et références, tome II (1991); vol. 7, Documents et écrits relatifs au chant syriaque maronite (1997). Série textes: vol. 5A, Les strophes-types syriaques, tome I. Les mètres poétiques du patriarche Étienne Duwayhi, introduction, traduction, commentaire et édition critique par P. Louis Hage (1986); vol. 5B, Les strophes-types syriaques, tome II, traduction et commentaire (1995); vol. 6, Les strophes-types syriaques, tome III, Analyse, classement et références (1996).

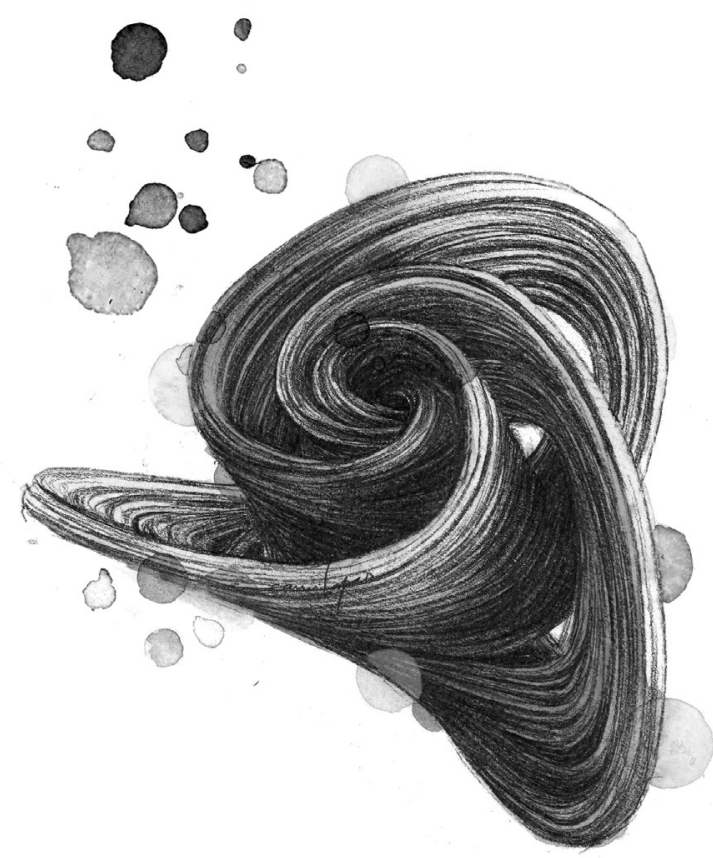

